\title{
Reaction mechanism of azoreductases suggests convergent evolution with quinone oxidoreductases
}

\author{
Ali Ryan*, Chan-Ju Wang*, Nicola Laurieri*, Isaac Westwood, Edith Sim ${ }^{凶}$ \\ Department of Pharmacology, University of Oxford, Mansfield Road, Oxford, OX1 3QT, UK \\ $\triangle$ Correspondence: edith.sim@pharm.ox.ac.uk \\ Received June 2, 2010 Accepted June 28, 2010
}

\begin{abstract}
Azoreductases are involved in the bioremediation by bacteria of azo dyes found in waste water. In the gut flora, they activate azo pro-drugs, which are used for treatment of inflammatory bowel disease, releasing the active component 5 -aminosalycilic acid. The bacterium $P$. aeruginosa has three azoreductase genes, paAzoR1, paAzoR2 and paAzoR3, which as recombinant enzymes have been shown to have different substrate specificities. The mechanism of azoreduction relies upon tautomerisation of the substrate to the hydrazone form. We report here the characterization of the $P$. aeruginosa azoreductase enzymes, including determining their thermostability, cofactor preference and kinetic constants against a range of their favoured substrates. The expression levels of these enzymes during growth of $P$. aeruginosa are altered by the presence of azo substrates. It is shown that enzymes that were originally described as azoreductases, are likely to act as NADH quinone oxidoreductases. The low sequence identities observed among NAD(P)H quinone oxidoreductase and azoreductase enzymes suggests convergent evolution.
\end{abstract}

KEYWORDS azoreductase, enzyme mechanism, $\mathrm{NAD}(\mathrm{P}) \mathrm{H}$ quinone oxidoreductase, enzyme characterisation, convergent evolution

\section{INTRODUCTION}

Dyes that contain an azo bond $(-\mathrm{N}=\mathrm{N}-)$ contribute significantly to world dye production (Stolz, 2001). Almost half of the dye used for dying fabrics does not bind to the fabric, and as a result, is lost in the effluent (Shore, 1995). As these dyes can be carcinogenic (Alves de Lima et al., 2007) their removal from the effluent is essential. This has driven investigations into both electrochemical reduction of azo dyes (Wang et al., $2010 \mathrm{~b}$ ), as well as the use of both aerobic and anaerobic bacteria for bioremediation (dos Santos et al., 2007; You et al., 2007).

Azo bonds are also found in some drugs including those used for the treatment of inflammatory bowel disease (IBD). Azo compounds have also been shown to be effective antibacterial (Farghaly and Abdalla, 2009) and antitumor (ElShafei et al., 2009) agents. Azo drugs for the treatment of IBD consist of a carrier compound linked via an azo bond to 5amino salycilic acid (5-ASA). 5-ASA if administered orally is rapidly removed from the digestive system and so it is modified with an inert carrier to prolong its action (Lichtenstein and Kamm, 2008). The azo bond is cleaved by intestinal bacteria (Peppercorn and Goldman, 1972) releasing 5-ASA.

Enzymes able to reduce an azo bond have been found in a number of species including Escherichia coli (Nakanishi et al., 2001), Pseudomonas aeruginosa (Wang et al., 2007), Enterococcus faecalis (Chen et al., 2004), Sinorhizobium meliloti (Ye et al., 2007), Saccharomyces cerevisiae (Sollner et al., 2007) and humans (Cui et al., 1995). These enzymes are flavin dependent oxidoreductases. Despite some of them sharing only minimal sequence identity, they have a common three dimensional fold (Fig. 1B) (Li et al., 1995; Ito et al., 2006; Wang et al., 2007; Ye et al., 2007; Binter et al., 2009).

The azoreductase reaction is proposed to be catalyzed via a Bi-Bi ping-pong mechanism (Nakanishi et al., 2001; Liu et al., 2008; Wang et al., 2010a), and we have indicated that the reaction is likely to occur via reduction of the hydrazone

\footnotetext{
"These authors contributed equally to the work.
} 
tautomer of the substrate, as identified from structural studies (Fig. 1A) (Ryan et al., 2010). The first reduction reaction is proposed to be followed by cleavage of the hydrazine intermediate via an acid or base catalyzed reaction (Fig. 1C). This cleavage releases a quinoneimine that is believed to be the substrate for the second reduction reaction (Fig. 1D).

A number of bacterial enzymes, classified as azoreductases, can also reduce quinones (Nakanishi et al., 2001; Chen et al., 2004; Liu et al., 2008; Binter et al., 2009). The mechanism for azo reduction that has been proposed (Ryan et al., 2010) explains the ability of azoreductases to reduce both azo and quinone substrates. It has been suggested that the major biological role of azoreductases is in the detoxification of quinones (Liu et al., 2008, 2009). These data suggest that azoreductases should be reclassified as $N A D(P) H$ quinone oxidoreductases (NQOs). NQOs have also been shown to be important for bacterial metabolism of some substrates, e.g., p-nitrophenol (Zhang et al., 2009). Even though mammalian NQOs have very low sequence identity with the bacterial enzymes (less than $15 \%$ between paAzoR1 and either human NQO1 or NQO2), they also reduce quinone and azo compounds (Cui et al., 1995). Recent evidence implicates the eukaryotic NQOs in the regulation of proteasomal degradation of tumor suppressor proteins (Sollner et al., 2009b; Alard et al., 2010). Therefore, further understanding of the relationship between azoreductases and $\mathrm{NAD}(\mathrm{P}) \mathrm{H}$ quinone oxidoreductases has wide implications in biology.

Three azoreductases have been identified in the genome of $P$. aeruginosa: these are termed paAzoR1 (PA0785), paAzoR2 (PA1962) and paAzoR3 (PA3223 (Wang et al., 2007)). The three enzymes have significantly different substrate specificities (Ryan et al., 2010), and we describe here a detailed enzymic characterization of these proteins, including the NADH quinone oxidoreductase activity of paAzoR3.

\section{RESULTS}

\section{Cofactor and hydride donor preference}

Absorbance spectra for each purified enzyme (Fig. 2) show that all three proteins have a typical flavoprotein signature: absorbance maxima shifted $7-10 \mathrm{~nm}$ bathochromically compared to that of flavin mononucleotide (FMN). The spectra each have a distinct shoulder at $\lambda_{\max }$ of $486 \mathrm{~nm}$ as an indication of binding of the flavin cofactor to the protein (Fig. 2) (Duurkens et al., 2007). The flavins are released as indicated by disappearance of the shoulder in the spectra upon denaturation of the protein and were identified as FMN via TLC (Supplemental Fig. 1).

paAzoR1 is unusual among azoreductases. Unlike many of these enzymes (Nakanishi et al., 2001; Deller et al., 2006), it can utilize both NADH and NADPH as electron donors with a preference for NADPH (Wang et al., 2007). Interestingly, this preference was reversed on mutation of Tyr131 to phenylalanine in the enzyme's active site (Wang et al., 2010a). In contrast to paAzoR1, which shows a preference for NADPH as the hydride donor, both paAzoR2 and paAzoR3 show a preference for NADH (Fig. 3A). paAzoR1 shows the same hydride donor preference when reducing the quinoneimine 2,6-dichloroindophenol (DCIP) as it does when reducing the azo substrate methyl red (Fig. 3B); however, the difference in quinone reductase activity with each of the two nicotinamide cofactors is smaller than what is observed when reducing azo substrates. In contrast paAzoR3 utilizes both $\mathrm{NADH}$ and NADPH with a preference for NADH of less than twofold when reducing methyl red, while the rate of reduction of DCIP with NADH is almost 20 times greater than with NADPH (Fig. 3C).

The reasons behind the nicotinamide cofactor preference remain unclear. Two crystal structures have been solved showing nicotinamide cofactors bound to flavodoxin-like proteins. The first structure showed rat NQO binding $\mathrm{NADP}^{+}$and has been withdrawn from the PDB (Li et al., 1995). The second structure shows NADH binding to EmoB from Mesorhizobium sp. BNC1 (PDB: 2VZJ) (Nissen et al., 2008). NADH binds EmoB in a solvent exposed cleft that extends from the solvent channel occupied by methyl red in the structure bound to paAzoR1. When the structure of EmoB is aligned with the paAzoR1, however, there are significant clashes between residue side chains and NADH; as a result, it is unlikely that NADH will bind in this cleft. The most possible binding position would be similar to what was modeled by Sollner et al. (2009a), where NADH binds in the same pocket of the active site as balsalazide (Fig. 1A).

\section{Enzyme stability}

Although $P$. aeruginosa lives in relatively mild conditions in soil and on human skin and in the intestinal flora, paAzoR1 has previously been shown to be thermostable with a melting temperature $\left(T_{m}\right)$ of approximately $55^{\circ} \mathrm{C}$ (Wang et al., 2007). Both paAzoR2 and paAzoR3 show significantly lower melting temperatures when compared to wild type paAzoR $1\left(40^{\circ} \mathrm{C}\right.$ and $50^{\circ} \mathrm{C}$, respectively, Fig. 4). Thermostability is not uncommon among azoreductases, e.g., B. subtilis $\left(T_{m}\right.$ $\left.86.5^{\circ} \mathrm{C}\right)$ and $\mathrm{S}$. cerevisiae $\left(\mathrm{T}_{\mathrm{m}} 60.2^{\circ} \mathrm{C}\right)$ determined via circular dichroism (Deller et al., 2006). When Tyr131 was mutated to Phe within the active site of paAzoR1 (equivalent residues are prolines in paAzoR2 and paAzoR3), it caused a significant shift in $\mathrm{T}_{\mathrm{m}}$ to approximately $42^{\circ} \mathrm{C}$ (Wang et al., 2010a). Oligomerisation state has previously been suggested as a possible reason for high thermostability (Deller et al., 2006), although later work on $B$. subtilis azoreductase disagreed (Binter et al., 2009). Preliminary data from analytical ultracentrifugation experiments on paAzoR2 and paAzoR3 (Wang, 2008) indicate they form dimeric and trimeric 


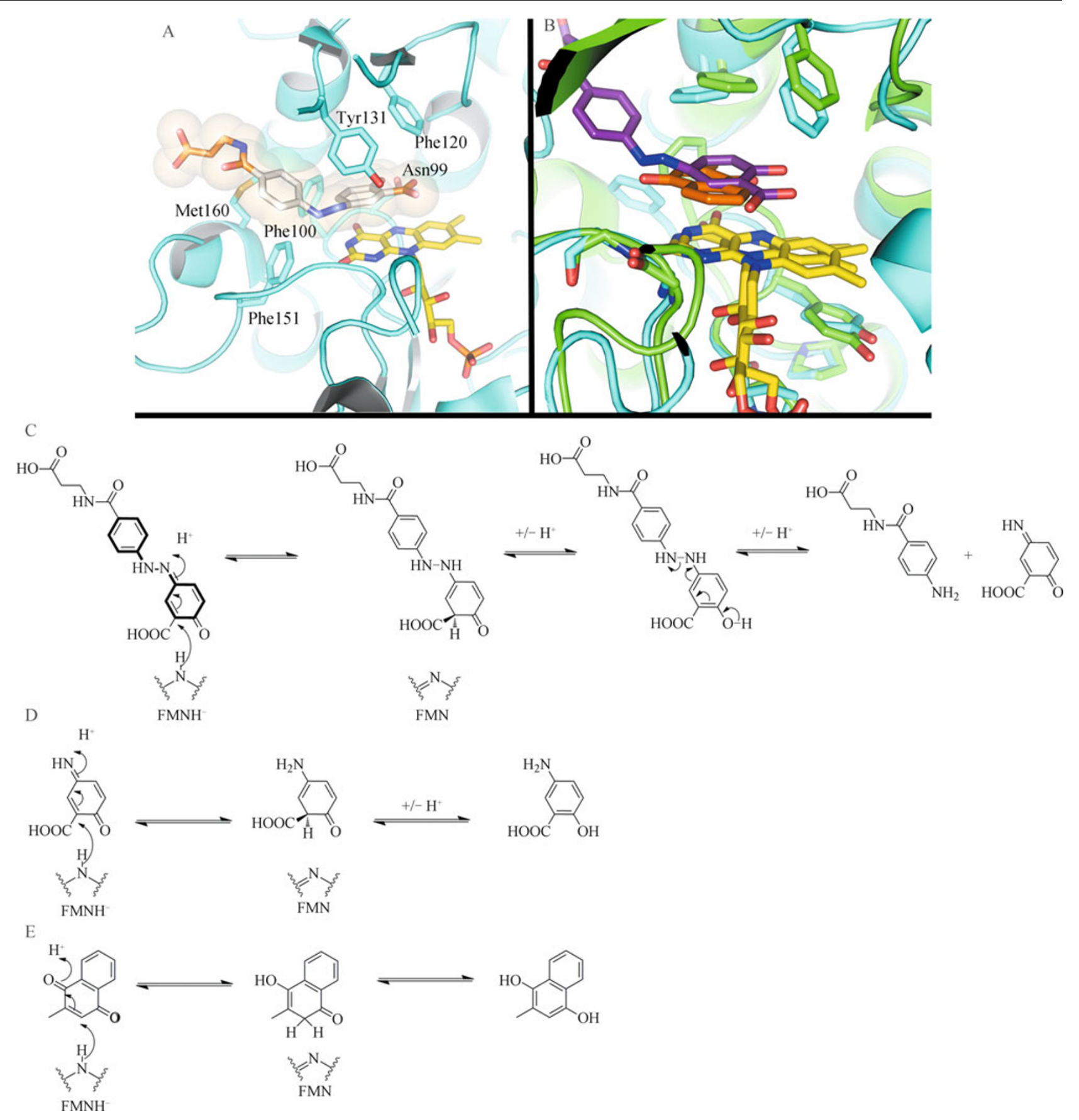

Figure 1. Proposed mechanism for balsalazide reduction by paAzoR1. (A) Structure of paAzoR1 binding the prodrug balsalazide (PDB: 3 LT5 (Ryan et al., 2010)). Balsalazide is shown in stick formation with a semi-transparent surface, the carbon atoms, which form its azobenzene core, are in white while all other carbon atoms are in orange. The FMN cofactor is in yellow. Important residues lining the active site are shown and labeled. The figure was prepared in PyMOL (version 1.1). (B) The panel shows human NQO2 with menadione bound (PDB: 2QR2) (Foster et al., 1999) aligned with the structure of paAzoR1 with balsalazide bound (PDB: 3LT5), with an overall RMSD of $2.2 \AA$ (overall sequence identity $11 \%$ ). paAzor1 is in green while human NQO2 is in turquoise. The balsalazide ligand is in purple while the menadione ligand is in orange and the flavin cofactors are in yellow (FAD in NQO1 and FMN in paAzoR1). Conserved residues lining the active site are shown. Structure alignment was carried out using secondary structure matching over 334 atoms in CCP4MG (version 2.3.0) (Potterton et al., 2004). (C) The panel shows the first round of reduction of the balsalazide hydrazone tautomer as proposed by Ryan et al. (2010). The azobenzene core of balsalazide is highlighted in bold in the first step of the reaction. This is followed by spontaneous cleavage of the hydrazine intermediate in an acid/base catalyzed reaction. (D) Proposed mechanism for the reduction of the quinoneimine produced by acid/base catalyzed N-N bond cleavage of the hydrazine intermediate of azo reduction. (E) Proposed mechanism for the reduction of menadione. This mechanism is based upon data from azo compounds. 
A

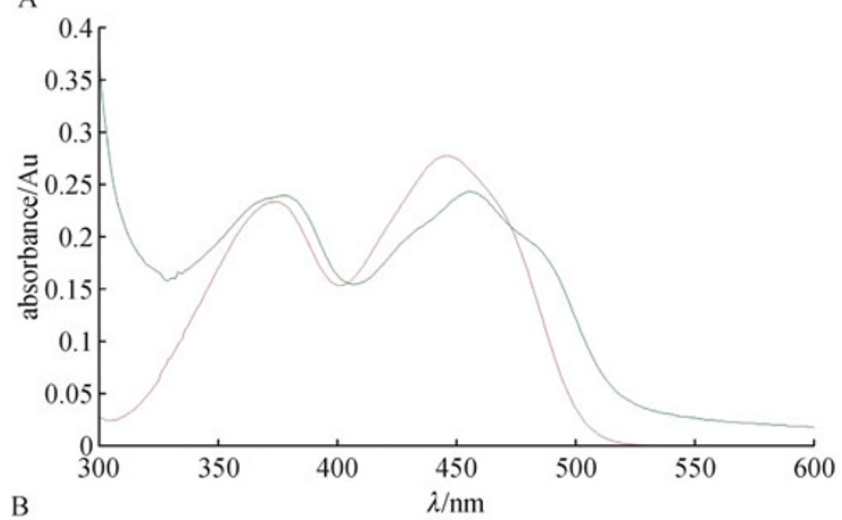

B

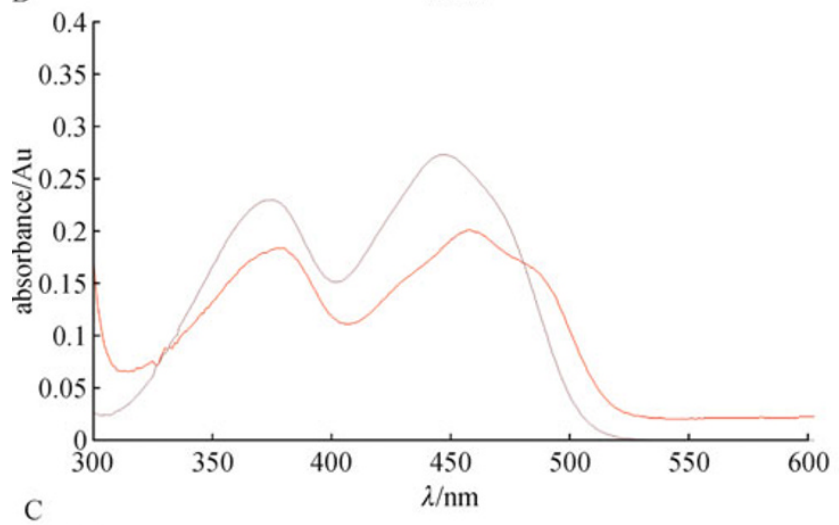

$\mathrm{C}$

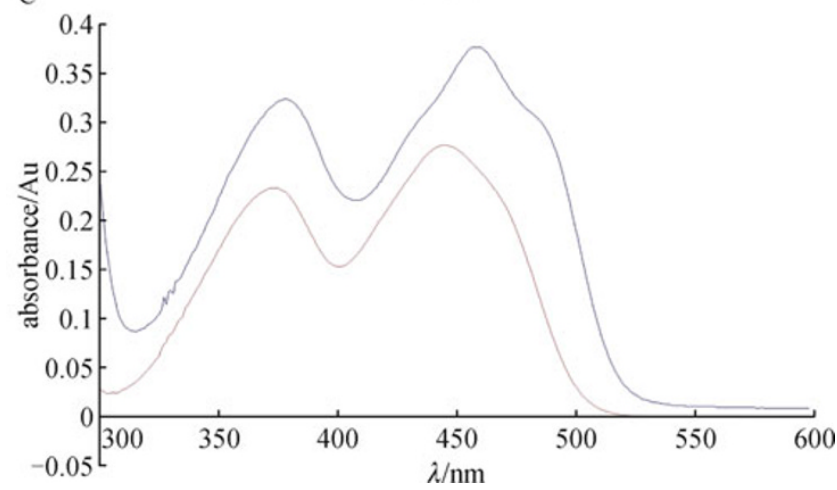

Figure 2. Absorption spectra of paAzoRs compared to soluble flavins. (A) UV/visible spectrum of paAzoR1 at $2 \mathrm{mg} / \mathrm{mL}$ in $20 \mathrm{mM}$ Tris- $\mathrm{HCl} \mathrm{pH} 8,0.3 \mathrm{M} \mathrm{NaCl}$. (B) UV/visible spectrum of paAzoR2 at $2 \mathrm{mg} / \mathrm{mL}$ in $20 \mathrm{mM}$ Tris- $\mathrm{HCl} \mathrm{pH} 8,0.3 \mathrm{M} \mathrm{NaCl}$. (C) UV/visible spectrum of paAzoR3 at $2 \mathrm{mg} / \mathrm{mL}$ in $20 \mathrm{mM}$ Tris- $\mathrm{HCl}$ $\mathrm{pH} 8,0.3 \mathrm{M} \mathrm{NaCl}$. In all panels, the FMN spectrum is shown in maroon.

assemblies, respectively, which supports the proposal that oligomerisation state may play a role in determining thermostability.

paAzoR1, paAzoR2 and paAzoR3 are tolerant of high salt concentrations showing greater than $40 \%$ maximal activity even at $\mathrm{NaCl}$ concentrations up to $2.5 \mathrm{M}$ (Fig. 5). In contrast to paAzoR2 and paAzoR3 whose peak activity is at $0.1 \mathrm{M} \mathrm{NaCl}$ (Fig. 5B and $5 \mathrm{C}$ ), peak activity for paAzoR 1 is around $1 \mathrm{M}$ $\mathrm{NaCl}$ (Fig. 5A). This high tolerance to salt may be due to

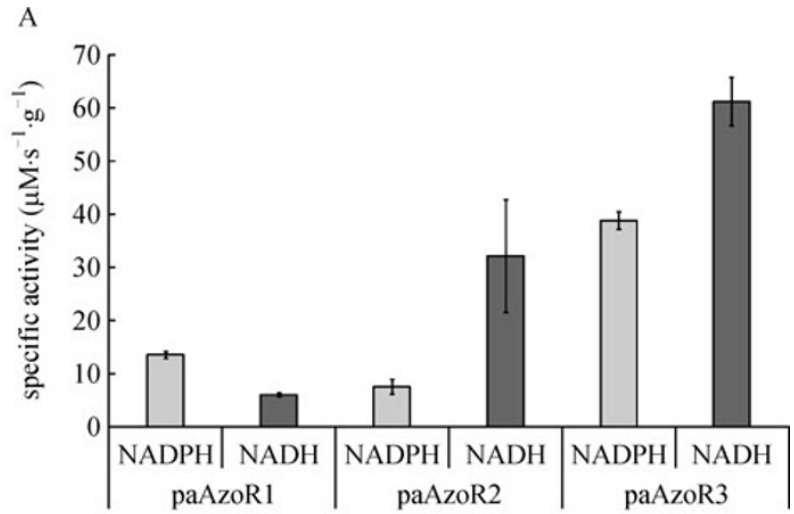

B
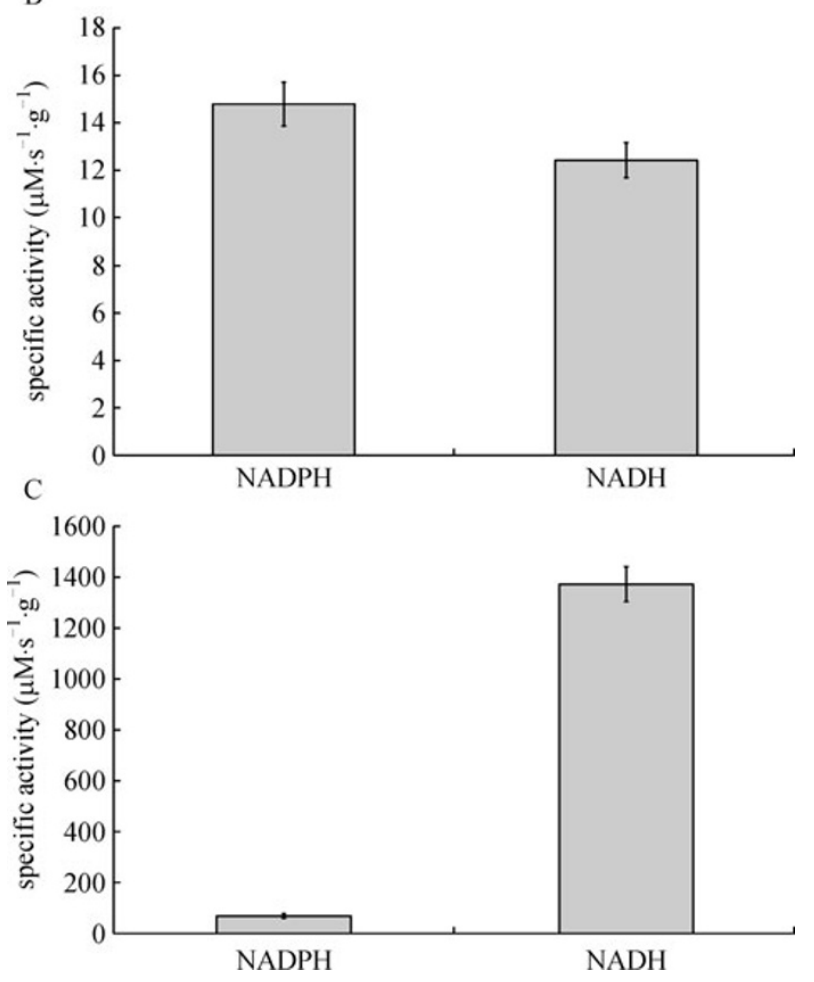

Figure 3. Nicotinamide preference of paAzoR1, paAzoR2 and paAzoR3. (A) Specific activity of paAzoRs with NADPH versus $\mathrm{NADH}$ as electron donor. $50 \mu \mathrm{M}$ methyl red, $0.5 \mathrm{mM}$ $\mathrm{NAD}(\mathrm{P}) \mathrm{H}$ were used as the substrates for all three azoreductases. (B) Specific activities of paAzoR1 with NADH versus $\mathrm{NADPH}$ as electron donor. $50 \mu \mathrm{M}$ DCIP and $0.5 \mathrm{mM} \mathrm{NAD}(\mathrm{P}) \mathrm{H}$ were used as substrates. $5 \mu \mathrm{g}$ of enzyme was used per reaction. (C) Specific activities of paAzoR3 with $0.5 \mathrm{mM}$ of $\mathrm{NADH}$ versus NADPH as electron donor. $50 \mu \mathrm{M}$ DCIP and $0.5 \mathrm{mM} N A D(P) H$ were used as substrates. $0.1 \mu \mathrm{g}$ of enzyme was used per reaction. All results are expressed as mean \pm standard deviation from three determinations.

stronger interactions at the predominantly hydrophobic oligmer interfaces. This high salt tolerance is advantageous for degradation of azo dyes, since salt concentrations can be as high as $2-3 \mathrm{M}$ in textile effluents (Environmental Protection Agency, 1997). 

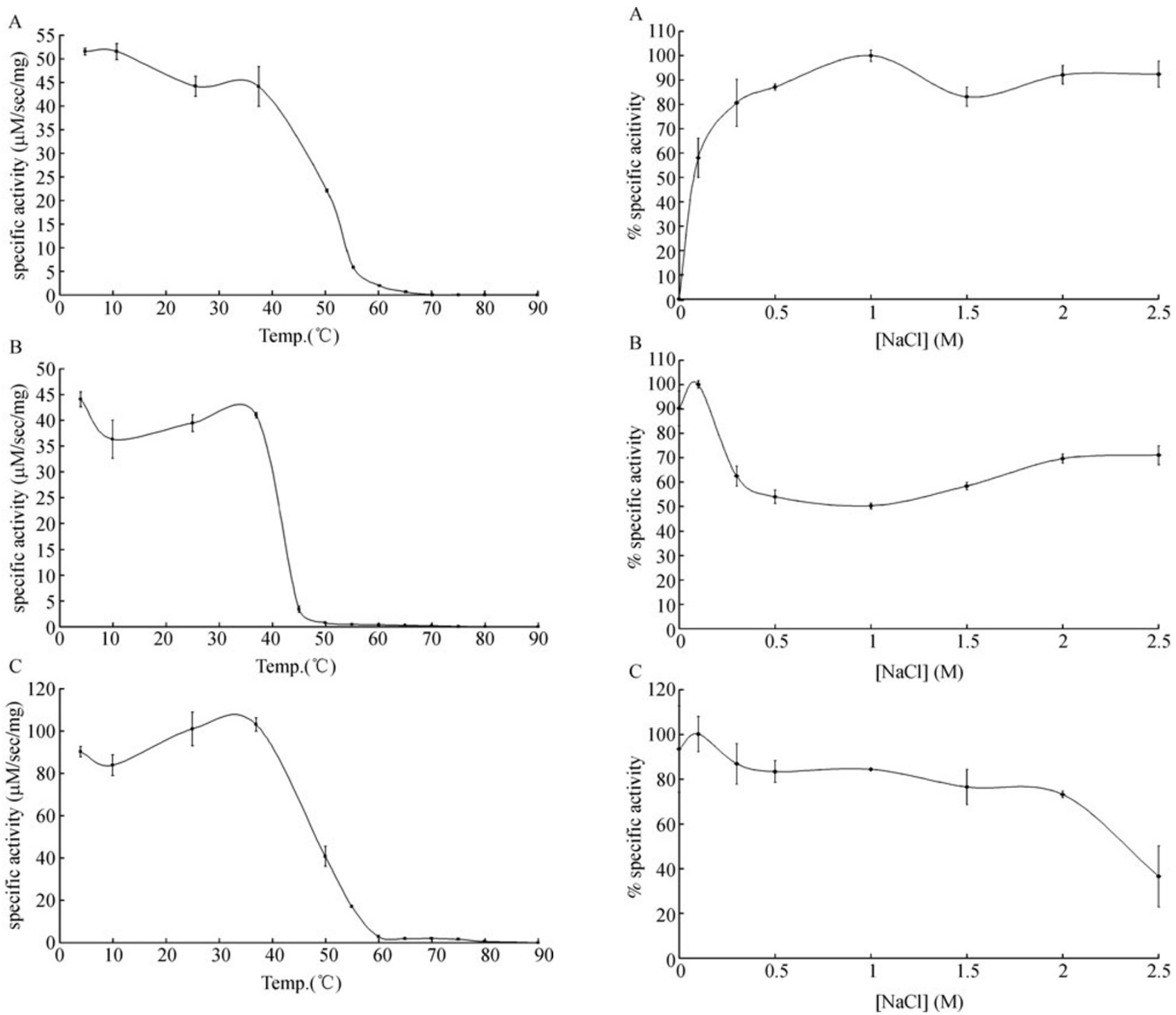

Figure 4. Thermostability of paAzoR1, paAzoR2 and paAzoR3. All three enzymes were tested for their thermostability after heating to a temperature of $25-100^{\circ} \mathrm{C}$ for $10 \mathrm{~min}$ before cooling on ice for $1 \mathrm{~min}$. The azoreductases were heated in the presence of $50 \mu \mathrm{M}$ methyl red $0.5 \mathrm{mM} \mathrm{NAD}(\mathrm{P}) \mathrm{H}$ were used as the substrate for all reactions. Results are expressed as mean \pm standard deviation from three determinations.

\section{Azo substrate kinetics}

The true values for the kinetic constants for all three azoreductases are shown in Table 1. One notable result is the variation in $K_{\mathrm{m}}$ values for the preferred hydride donor. In the cases of paAzoR2 and paAzoR3 the $K_{m}$ for NADH is $525 \mu \mathrm{M}$ and $298 \mu \mathrm{M}$, respectively; in contrast, the $K_{\mathrm{m}}$ of paAzoR1 for NADPH is over $10 \mathrm{mM}$. This suggests that the activity of the enzymes may be tied to the redox state of the cell. It has also been proposed that the in vivo role of human

Figure 5. The effect of ionic strength on enzymic activity of azoreductases (A) paAzoR1, (B) paAzoR2 and (C) paAzoR3. The activity of each enzyme was measured in $20 \mathrm{mM}$ Tris- $\mathrm{HCl}$ $\mathrm{pH} 8$ buffered solutions containing $0-2.5 \mathrm{M} \mathrm{NaCl}$. Methyl red was used as the substrate in all three cases. Results are expressed as a percentage of the maximum observed specific activity for each enzyme. Results are expressed as mean \pm standard deviation from three determinations.

NQO1 is in regulating the intracellular redox state (Long et al., 2002).

During assays for paAzoR1 activity against methyl red, substrate inhibition was observed at methyl red concentrations of $>80 \mu \mathrm{M}$. The reason for this is believed to be the low affinity of paAzoR1 for NADPH, as the $K_{\mathrm{m}}$ for methyl red is $\sim 30$ folds lower than for NADPH. No substrate inhibition was observed with balsalazide; thus, although the binding of methyl red is significantly tighter than for balsalazide (greater 
Table 1 Kinetic values of paAzoRs

\begin{tabular}{|c|c|c|c|c|}
\hline paAzoR1 & methyl red & sulfasalazine & balsalazide & $\mathrm{NADPH}^{\mathrm{a}}$ \\
\hline$V_{\max }\left(\mu \mathrm{M} \cdot \mathrm{s}^{-1} \cdot \mathrm{mg}\right.$ protein $\left.^{-1}\right)$ & 953 & 508 & 3963 & \\
\hline$K_{m}(\mu \mathrm{M})$ & 260 & 1118 & 2447 & 10262 \\
\hline$k_{\text {cat }}\left(\mathrm{s}^{-1}\right)$ & 4.8 & 2.6 & 20.0 & \\
\hline specificity constant $\left(\mathrm{mM}^{-1} \cdot \mathrm{s}^{-1}\right)$ & 18.5 & 2.3 & 8.2 & 0.5 \\
\hline paAzoR2 & methyl red & amaranth & ponceau BS & $\mathrm{NADH}^{\mathrm{a}}$ \\
\hline$V_{\max }\left(\mu \mathrm{M} \cdot \mathrm{s}^{-1} \cdot \mathrm{mg}\right.$ protein $\left.^{-1}\right)$ & 230 & 180 & 157 & \\
\hline$K_{m}(\mu \mathrm{M})$ & 303 & 380 & 55 & 525 \\
\hline$k_{\text {cat }}\left(\mathrm{s}^{-1}\right)$ & 1.0 & 0.8 & 0.7 & \\
\hline specificity constant $\left(\mathrm{mM}^{-1} \cdot \mathrm{s}^{-1}\right)$ & 3.3 & 2.1 & 12.7 & 1.9 \\
\hline paAzoR3 & methyl red & amaranth & olsalazine & $\mathrm{NADH}^{\mathrm{a}}$ \\
\hline$V_{\max }\left(\mu \mathrm{M} \cdot \mathrm{s}^{-1} \cdot \mathrm{mg}\right.$ protein $\left.^{-1}\right)$ & 2395 & 96 & 42 & \\
\hline$K_{m}(\mu \mathrm{M})$ & 983 & 220 & 341 & 298 \\
\hline$k_{\text {cat }}\left(\mathrm{s}^{-1}\right)$ & 11.6 & 0.5 & 0.2 & \\
\hline specificity constant $\left(\mathrm{mM}^{-1} \cdot \mathrm{s}^{-1}\right)$ & 11.8 & 2.3 & 0.6 & 38.9 \\
\hline
\end{tabular}

than 9:1 difference), balsalazide shows a markedly higher $V_{\text {max }}$ (more than 4:1).

Azobenzene was tested as a substrate for paAzoR1 and paAzoR3. In contrast to the other azo substrates, azobenzene has neither hydroxyl nor amine groups as substituents on the aromatic ring (Supplemental Fig. 2A); thus, it cannot form a hydrazone tautomer. Even using up to $20 \mu \mathrm{g}$ of either paAzoR1 or paAzoR3 in the reaction, there was no reduction of azobenzene with either NADH or NADPH as the hydride donor. Azobenzene is significantly smaller than balsalazide (Fig. 1A); therefore, it can easily be accommodated by the active site. These data support the proposal that formation of a hydrazone tautomer is essential for azo compounds to be reduced by this class of enzymes.

\section{Expression of azoreductases during growth on azo compounds}

Colonies of $P$. aeruginosa were grown in LB media containing one of the following drugs: balsalazide, sulfasalazine, olsalazine and 5-ASA, or dyes methyl red and ponceau BS. mRNA levels were monitored for each of the azoreductases via Q-PCR (Fig. 6).

In media containing methyl red, the levels of paAzoR2 mRNA increased by a factor of approximately 50 . Growth on methyl red also caused a significant (3.5 fold) increase in paAzoR3 mRNA levels. Interestingly growth on methyl red had the opposite effect on paAzoR1 whose mRNA levels were reduced by more than $50 \%$.

The other substrate where a significant increase was observed was growth on balsalazide, where again paAzoR2 mRNA levels showed the most significant increase ( 2.5 fold).
mRNA levels for paAzoR1 also increased but to a less extent (1.5 fold).

More significant increases in azoreductase mRNA levels have been described in $E$. coli and $B$. subtilis in the presence of quinones (Töwe et al., 2007; Liu et al., 2009), which is compatible with the proposal that quinones are the primary physiologic substrate for azoreductases.

\section{NAD(P)H quinone oxidoreductase activity}

We previously demonstrated that paAzoR 1 is able to reduce the quinoneimine substrate DCIP (Supplemental Fig. 2B) (Ryan et al., 2010). This reduction has now been studied in more detail in the cases of both paAzoR1 and paAzoR3. The ability of these enzymes to reduce the quinone substrate menadione (2-methyl-1,4-napthoquinone, Supplemental Fig. 2C) was also evaluated.

The $K_{\mathrm{m}}$ value for DCIP with paAzoR1 is similar to those obtained for azo compounds: $85.3 \pm 3.6 \mu \mathrm{M}$ for DCIP compared to $98.6 \mu \mathrm{M}$ for balsalazide (Wang et al., 2010a). The $V_{\max }$ value is similar to those observed for azo substrates: $V_{\text {max }}=0.53 \pm 0.01 \mu \mathrm{M} / \mathrm{s}$ for DCIP compared to $0.81 \mu \mathrm{M} / \mathrm{s}$ for balsalazide (Wang et al., 2010a). The specific activity for DCIP was however 3.5 fold higher than for balsalazide: 106 $\mu \mathrm{M} \cdot \mathrm{s}^{-1} \cdot \mathrm{mg}^{-1}$ for DCIP compared to $29.7 \mu \mathrm{M} \cdot \mathrm{s}^{-1} \cdot \mathrm{mg}^{-1}$ for balsalazide (Ryan et al., 2010).

In the case of paAzoR3, DCIP is reduced at least 30 times faster than methyl red. The maximum activity, which could be measured was at $18.75 \mu \mathrm{M}$ DCIP, and is equivalent to a specific activity $\sim 3220 \mu \mathrm{M} \cdot \mathrm{s}^{-1} \cdot \mathrm{mg}^{-1}$ ( $>30$ times that observed for methyl red the best azo substrate for paAzoR3) (Ryan et al., 2010). Due to substrate inhibition of the enzyme, it was not 

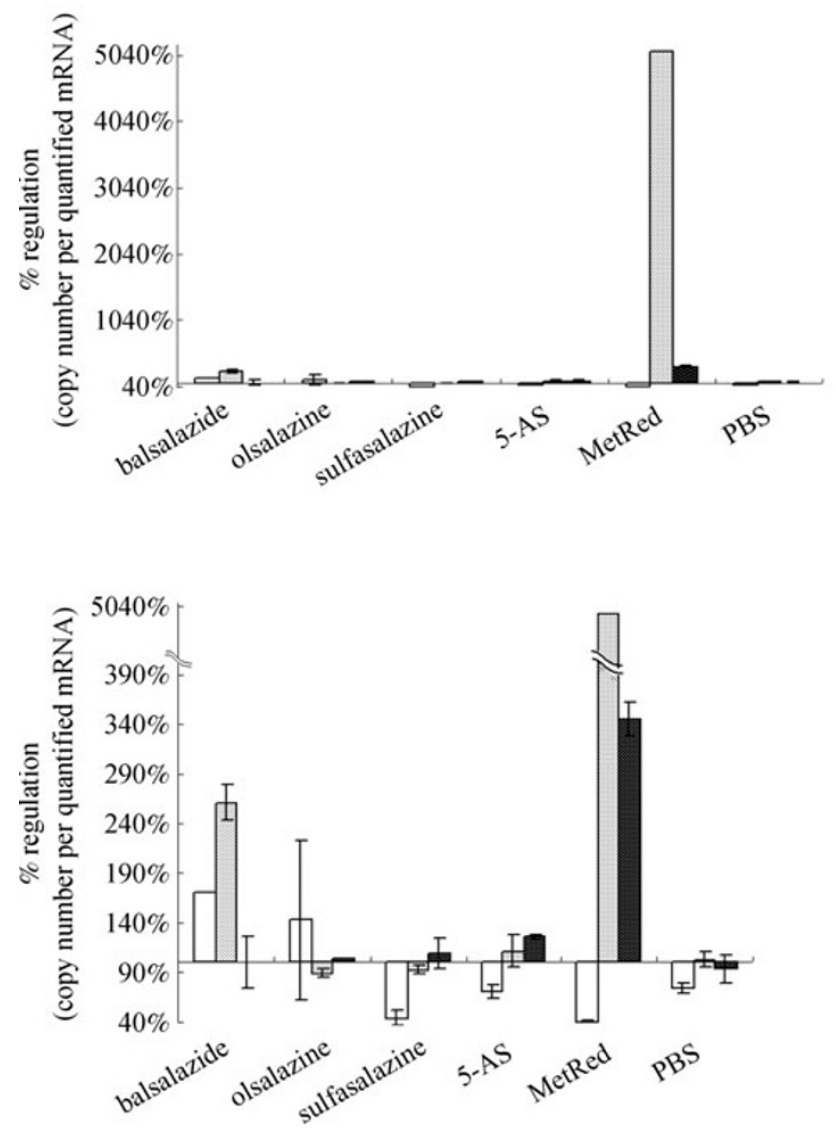

Figure 6. Regulation of paazor gene expressions in the presence of azo substrates. mRNA levels for the three azoreductases were measured after growth on media containing $1 \mathrm{mM}$ azo substrate or 5-ASA. mRNA levels were quantified via RT-PCR using levels of $r p o D$ as a standard. The lower panel shows an expanded view of the smaller changes in azoreductase expression. Ponceau BS is abbreviated to PBS in this figure. Results are expressed as mean \pm standard deviation from two determinations.

possible to determine the $K_{\mathrm{m}}$ of DCIP (Fig. 7); however, the $K_{\mathrm{m}}$ is likely to be less than $20 \mu \mathrm{M}$.

Menadione was also chosen as a test substrate as it is a substrate of azoreductases from E. coli (Nakanishi et al., 2001), E. faecalis (Chen et al., 2004) and R. sphaeroides (Liu et al., 2008). The structure of menadione bound to human NQO2 (PDB 2QR2) (Foster et al., 1999) is shown in Fig. 1B overlaid onto the structure of paAzoR1. The proposed mechanism for menadione reduction, based on data from azo compounds, is shown in Fig. 1E. paAzoR1 did not show activity with menadione even when up to $20 \mu \mathrm{g}$ of enzyme was used in the assay using either NADH or NADPH as hydride donor. These data reinforce the concept that paAzoR1 has a strong preference for reduction of single ring substrates, e.g., methyl red (Supplemental Fig. 2D), rather than naphthol containing substrates, e.g., ponceau BS

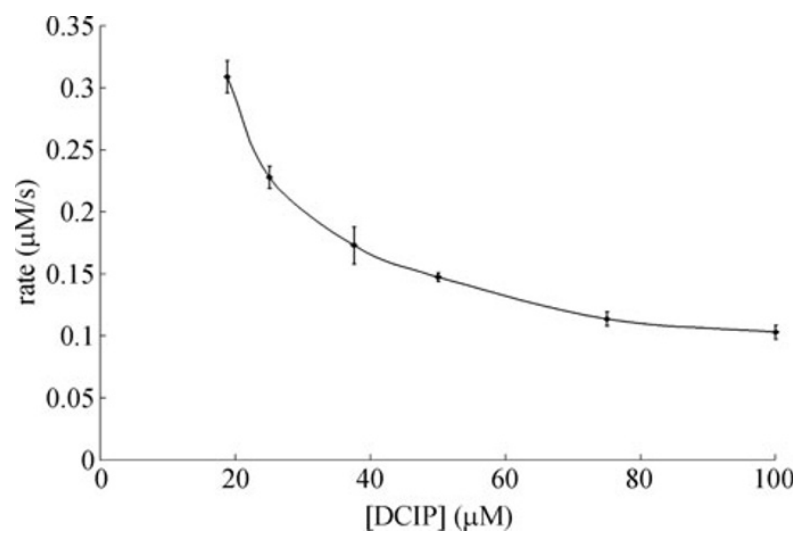

Figure 7. Rate of reduction of DCIP by paAzoR3. Rates were determined using $0.1 \mu \mathrm{g}$ of paAzoR3 per well $0.5 \mathrm{mM}$ $\mathrm{NADH}$. Linear rates were measured over $30 \mathrm{~s}$. Results are expressed as mean \pm standard deviation from three determinations.

(Supplemental Fig. 2E) (Ryan et al., 2010).

On the other hand, paAzoR3, which reduced naphthol containing azo substrates, showed a high affinity for menadione $\left(K_{\mathrm{m}}<7.5 \mu \mathrm{M}\right)$ with $\mathrm{NADH}$ as a proton donor. The specific activity for the reduction of menadione was calculated as $910 \mu \mathrm{M} \cdot \mathrm{s}^{-1} \cdot \mathrm{mg}^{-1}$ (ten times the specific activity observed with methyl red). When NADPH was used as the proton donor no activity was observed. This shows that paAzoR3 is an NADH quinone oxidoreductase as well as an azoreductase.

\section{DISCUSSION}

The physiologic role of bacterial azoreductases has remained an unanswered question although recent work has suggested they may be involved in the detoxification of quinones (Liu et al., 2009). The accepted mechanism for azoreduction was unable to explain how both azo and quinone substrates are reduced by the same enzyme. However, we have resolved the apparent contradiction by proposing a novel mechanism for azoreduction (Fig. 1C and 1D) (Ryan et al., 2010). The inability of paAzoR1 and paAzoR3 to reduce azobenzene, a substrate unable to form the hydrazone tautomer required for the proposed mechanism, lends strong support to this mechanism. The ability of both paAzoR1 and paAzoR3 to reduce DCIP, an analog of the proposed quinoneimine substrates, also supports the proposed mechanism. The high affinity of paAzoR3 for the quinone, menadione and its ability to reduce it at a higher rate than azo compounds further support its role as an NADH quinone oxidoreductase.

A number of therapeutic compounds, including antitumor drugs such as mitomycin C (Tomasz and Palom, 1997), are quinone based agents that are activated in humans upon reduction by azoreductase-like proteins. A better under- 
standing of the enzymes that activate these drugs in the body may allow the design of more selective compounds. There is an extensive history of using information from bacterial enzymes to understand the interactions of human enzymes with xenobiotics, e.g., cytochrome P450s (Poulos et al., 1985) and more recently the arylamine $\mathrm{N}$-acetyl transferase, enzymes (Sinclair et al., 2000). The use of a series of bacterial enzymes has proved particularly instructive (Lewis, 2001; Sandy et al., 2005).

This manuscript describes the first detailed characterization of a series of azoreductases from the same organism. Due to the sequence diversity which is observed among proteins which adopt the flavodoxin-like fold there may be further azoreductase-like enzymes in $P$. aeruginosa that have yet to be characterized, e.g., pa0949 from $P$. aeruginosa shares only $11 \%$ sequence identity with paAzoR 1 , but shares approximately $40 \%$ sequence identity with tryptophan repressor binding protein $A(W r b A)$ from $E$. coli which has an azoreductase activity (Patridge and Ferry, 2006). The number of azoreductase-like enzymes and the diversity of their substrates may suggest that they play more than one physiologic role and provides insight into the interactions of the human structural and functional homologs with xenobiotics. This is important for their interactions with not only drugs but also environmental toxins.

The sequence diversity among flavodoxin-like proteins is possible in part due to the non-sequence specific manner in which the flavin binding cradle operates (Ryan et al., 2010). Diverse enzymes belonging to this class of enzymes have been identified within organisms but also among different

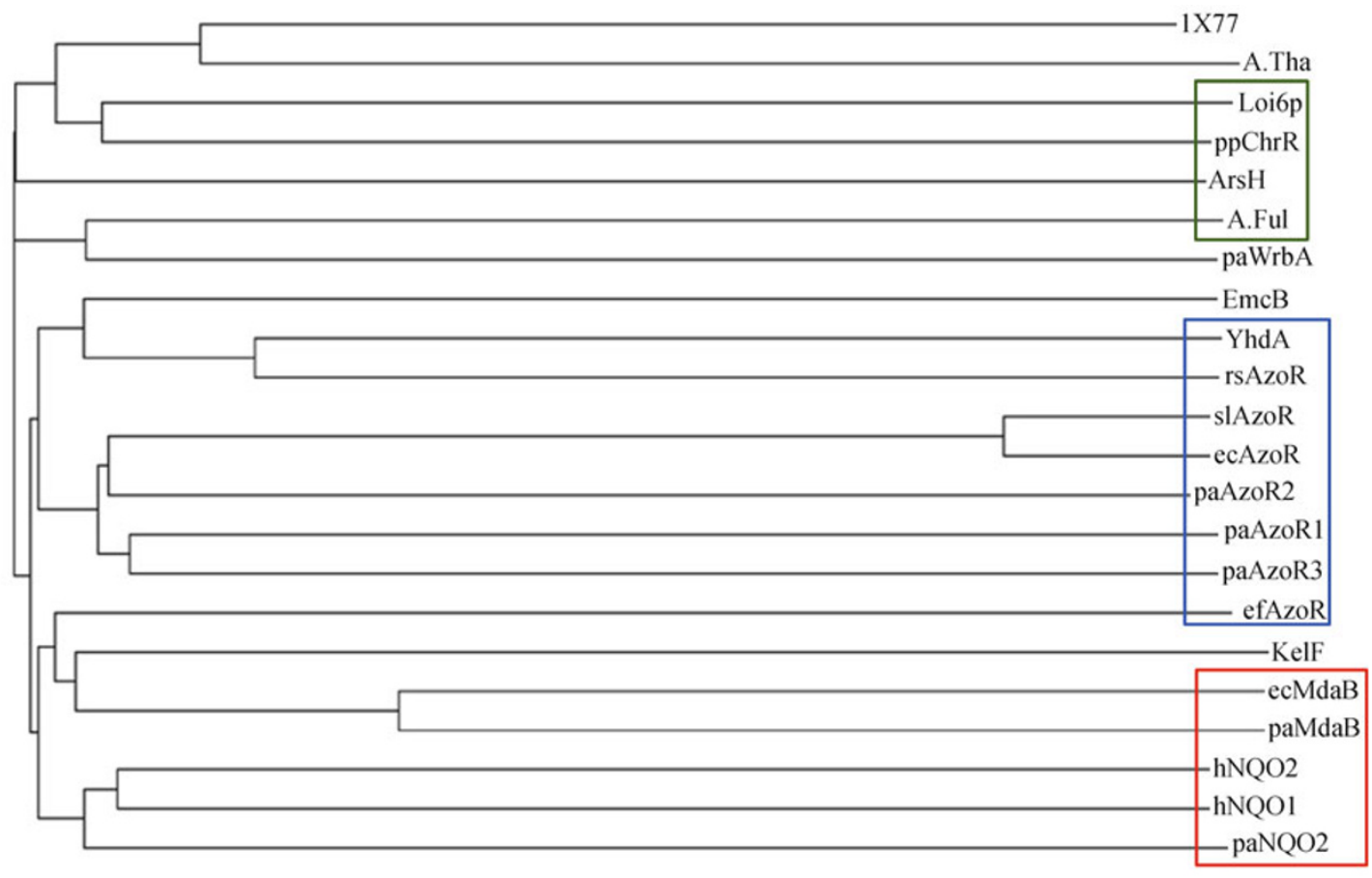

Figure 8. Phylogenetic tree showing the evolution of a group of $\mathbf{2 0}$ two flavodoxin enzymes. The dendrogram was generated via the neighbor-joining method as part of the Expresso multiple sequence alignment algorithm (Armougom et al., 2006). Bacterial azoreductases are highlighted boxed in blue, heavy metal reductases are boxed in green and mammalian NQO related enzymes are boxed in red. Branch length is proportional to the predicted time since the enzymes diverged. The sequences for paAzoR1, 2 and 3 are azoreductases from $P$. aeruginosa. ecAzoR, rsAzoR, stAzoR and efAzoR are azoreductases from $E$. coli (Nakanishi et al., 2001), R. sphaeroides (Bin et al., 2004), S. typhimurium (Zhang et al., 2004) and E. faecalis (Chen et al., 2004). paWrbA is the sequence for WrbA from $P$. aeruginosa(Gorman and Shapiro, 2005), KefF is an enzyme from E. coli (Roosild et al., 2009), ecMdaB and paMdaB are the mediator of drug activity B from E. coli (Adams and Jia, 2006) and $P$. aeruginosa respectively, YhdA is an enzyme from B. subtilis (Binter et al., 2009), EmoB is an enzyme fromMesorhizobiums p. BNC1 (Nissen et al., 2008), ArsH is an enzyme from S. meliloti (Ye et al., 2007), Lot6p is an enzyme from S. cerevisiae (Liger et al., 2004), ppChrR is the chromate reductase from $P$. putida (Ackerley et al., 2004). 1 X77 is an NADPH dependent oxidoreductase from $P$. aeruginosa (Agarwal et al., 2006), hNQO1 and hNQO2 are human NQOs (Foster et al., 1999; Li et al., 1995), A. tha is the sequence for an $\mathrm{NAD}(\mathrm{P}) \mathrm{H}$ quinone reductase from $A$. thaliana (Sparla et al., 1999). A. ful is the sequence of WrbA from the archaean A. fulgidus (Patridge and Ferry, 2006). 
kingdoms. These are connected by common activities that link proteins from archaea, eukaryotes and prokaryotes. A phylogenetic tree has been generated using the neighborjoining method for the amino acid sequence of flavodoxin like proteins (Fig. 8) (Armougom et al., 2006). This tree clearly shows the division of the enzymes into three groups: bacterial azoreductases, enzymes that are related to the mammalian NQO enzymes and heavy metal reducing enzymes. What is particularly interesting is that these groups cross kingdoms, especially in the case of the heavy metal enzymes, including plants, yeast, and bacterial and archaeal members. This indicates that evolution does not follow the standard species lines and may suggest convergent evolution.

\section{MATERIALS AND METHODS}

Chemicals were purchased from Sigma-Aldrich, unless otherwise stated. DNA primers were also synthesized by Sigma-Aldrich (Supplemental Table 1). The $P$. aeruginosa PAO1 Washington strain was kindly provided by $\mathrm{Dr}$ Gail Preston, Department of Plant Sciences, University of Oxford, UK.

paAzoR1, paAzoR2 and paAzoR3 were expressed and purified as described previously (Wang et al., 2007). Enzyme characterization was carried out as detailed previously (Wang et al., 2007). 2,6dichlorindophenol (DCIP) reduction was measured via its absorbance at $595 \mathrm{~nm}$ and an extinction coefficient of $14,690 \mathrm{M}^{-1} \cdot \mathrm{cm}^{-1}$ was used. Reduction of menadione was measured, as described previously (Nakanishi et al., 2001), via monitoring the oxidation of $\mathrm{NAD}(\mathrm{P}) \mathrm{H}$ (absorbance at $340 \mathrm{~nm}$ ). To measure accurately rates for menadione and azobenzene reduction $0.5 \mu \mathrm{g}$ of enzyme was used per well, $150 \mu \mathrm{M} \mathrm{NAD}(\mathrm{P}) \mathrm{H}$ and $30-7.5 \mu \mathrm{M}$ substrate in a total volume of $100 \mu \mathrm{L}$ of $20 \mathrm{mM}$ Tris- $\mathrm{HCl}, \mathrm{pH} 8.0$, containing $100 \mathrm{mM} \mathrm{NaCl}$ per well of a $96-$ well plate. Thermostability assays were carried out as described previously (Wang et al., 2010a), via measurement of enzyme activity versus methyl red after ten minutes incubation at defined temperatures.

\section{Quantitative RT-PCR}

Cultures of $P$. aeruginosa PAO1 were grown in LB medium containing $1 \mathrm{mM}$ azo compound or 5-ASA to an absorbance at $600 \mathrm{~nm}$ of 1.0 . A sample $(5 \mathrm{~mL})$ of each culture was removed RNA was extracted using an RNeasy Mini kit (Qiagen) followed by treatment with additional RNase-free DNase (Qiagen) to remove contaminating DNA. The concentration of RNA from each sample was adjusted to the same value for cDNA synthesis using a QuantiTect Reverse Transcription kit (Qiagen). Primers used in this study (RQ-785, RQ-962, RQ-223, rpoD, forward and reverse) were specifically designed to give PCR products around 150 base pair (Supplemental Table 1). Expression levels of paazor1, paazor2 and paazor 3 were detected by quantitative RT-PCR analysis with a LightCycler (Roche) in comparison with expression of a $P$. aeruginosa $\sigma$ factor rpoD (Savli et al., 2003). Each amplification mixture $(10 \mu \mathrm{L}$ QuantiTect $2 \times$ SYBR Green PCR master mix (Qiagen), $0.5 \mu \mathrm{g}$ cDNA, $0.5 \mu \mathrm{M}$ suitable primers and sterile water in a total $20 \mu \mathrm{L}$ ) was subjected to the following thermo-cycling program: one cycle of $95^{\circ} \mathrm{C}$ for $15 \mathrm{~min}$ to activate the HotStart Taq DNA polymerase; 40 cycles of denaturation $\left(94^{\circ} \mathrm{C}\right.$ for $\left.15 \mathrm{~s}\right)$, annealing $\left(60^{\circ} \mathrm{C}\right.$ for $\left.20 \mathrm{~s}\right)$, extension $\left(72^{\circ} \mathrm{C}\right.$ for $\left.15 \mathrm{~s}\right)$ and data acquisition $\left(65^{\circ} \mathrm{C}\right.$ for $5 \mathrm{~s})$. All reactions were performed in duplicate.

In all PCR studies, RNA concentration was determined by measuring the absorbance at $260 \mathrm{~nm}$ and the integrity was confirmed by $1 \%$ agarose gel electrophoresis. Absence of DNA contamination was confirmed by running duplicate samples without reverse transcriptase as a negative control.

\section{Thin layer chromatography}

Thin layer chromatography (TLC) analysis (Sherma, 2003) was performed on a $0.1 \mathrm{~mm}$ thick silica gel-coated glass plate (Merck) using the mobile solvent system: tertiary amyl alcohol/formic acid/ water (3:1:1, v/V) (Fetzner et al., 1992). Flavin co-factor was extracted from the protein component by denaturation $\left(95^{\circ} \mathrm{C}, 10 \mathrm{~min}\right)$ and centrifugation (10,000 g, $5 \mathrm{~min}$ ). Flavins were detected by direct visual observation of their yellow color. Authentic FMN, FAD and riboflavin were prepared in $20 \mathrm{mM}$ Tris- $\mathrm{HCl} \mathrm{pH} 8.0$ buffer as standards.

\section{ABBREVIATIONS}

5-ASA, 5-amino salycilate; DCIP, 2,6-dichloroindophenol; IBD, inflammatory bowel disease; FMN, Flavin mononucleotide; LB, Luria Bertani; NQOs, NAD(P)H quinone oxidoreductases; $T_{m}$, melting temperature; paAzoR, Pseudomonas aeruginosa azoreductase; WrbA, tryptophan repressor binding protein

\section{REFERENCES}

Ackerley, D.F., Gonzalez, C.F., Park, C.H., Blake, R. 2nd, Keyhan, M., and Matin, A. (2004). Chromate-reducing properties of soluble flavoproteins from Pseudomonas putida and Escherichia coli. Appl Environ Microbiol 70, 873-882.

Adams, M.A., and Jia, Z. (2006). Modulator of drug activity B from Escherichia coli: crystal structure of a prokaryotic homologue of DT-diaphorase. J Mol Biol 359, 455-465.

Agarwal, R., Bonanno, J.B., Burley, S.K., and Swaminathan, S. (2006). Structure determination of an FMN reductase from Pseudomonas aeruginosa PA01 using sulfur anomalous signal. Acta Crystallogr D Biol Crystallogr 62, 383-391.

Alard, A., Fabre, B., Anesia, R., Marboeuf, C., Pierre, P., Susini, C., Bousquet, C., and Pyronnet, S. (2010). NAD(P)H quinoneoxydoreductase 1 protects eukaryotic translation initiation factor $4 \mathrm{GI}$ from degradation by the proteasome. Mol Cell Biol 30, 1097-1105.

Alves de Lima, R.O., Bazo, A.P., Salvadori, D.M.F., Rech, C.M., de Palma Oliveira, D., and de Aragão Umbuzeiro, G. (2007). Mutagenic and carcinogenic potential of a textile azo dye processing plant effluent that impacts a drinking water source. Mutat Res 626, 53-60.

Armougom, F., Moretti, S., Poirot, O., Audic, S., Dumas, P., Schaeli, B., Keduas, V., and Notredame, C. (2006). Expresso: automatic incorporation of structural information in multiple sequence alignments using 3D-Coffee. Nucleic Acids Res 34, W604-608.

Bin, Y., Jiti, Z., Jing, W., Cuihong, D., Hongman, H., Zhiyong, S., and Yongming, B. (2004). Expression and characteristics of the gene encoding azoreductase from Rhodobacter sphaeroides AS1.1737. FEMS Microbiol Lett 236, 129-136.

Binter, A., Staunig, N., Jelesarov, I., Lohner, K., Palfey, B.A., Deller, S., Gruber, K., and Macheroux, P. (2009). A single intersubunit salt 
bridge affects oligomerization and catalytic activity in a bacterial quinone reductase. FEBS J 276, 5263-5274.

Chen, H., Wang, R.F., and Cerniglia, C.E. (2004). Molecular cloning, overexpression, purification, and characterization of an aerobic FMN-dependent azoreductase from Enterococcus faecalis. Protein Expr Purif 34, 302-310.

Cui, K., Lu, A.Y., and Yang, C.S. (1995). Subunit functional studies of $\mathrm{NAD}(\mathrm{P}) \mathrm{H}$ :quinone oxidoreductase with a heterodimer approach. Proc Natl Acad Sci U S A 92, 1043-1047.

Deller, S., Sollner, S., Trenker-El-Toukhy, R., Jelesarov, I., Gübitz, G. M., and Macheroux, P. (2006). Characterization of a thermostable NADPH:FMN oxidoreductase from the mesophilic bacterium Bacillus subtilis. Biochemistry 45, 7083-7091.

dos Santos, A.B., Cervantes, F.J., and van Lier, J.B. (2007). Review paper on current technologies for decolourisation of textile wastewaters: perspectives for anaerobic biotechnology. Bioresour Technol 98, 2369-2385.

Duurkens, R.H., Tol, M.B., Geertsma, E.R., Permentier, H.P., and Slotboom, D.J. (2007). Flavin binding to the high affinity riboflavin transporter RibU. J Biol Chem 282, 10380-10386.

El-Shafei, A., Fadda, A.A., Khalil, A.M., Ameen, T.A., and Badria, F.A. (2009). Synthesis, antitumor evaluation, molecular modeling and quantitative structure-activity relationship (QSAR) of some novel arylazopyrazolodiazine and triazine analogs. Bioorg Med Chem 17, 5096-5105.

Environmental Protection Agency. (1997). Profile of the Textile Industry. In Sector Notebook Project (Washington, DC).

Farghaly, T.A., and Abdalla, M.M. (2009). Synthesis, tautomerism, and antimicrobial, anti-HCV, anti-SSPE, antioxidant, and antitumor activities of arylazobenzosuberones. Bioorg Med Chem 17, 8012-8019.

Fetzner, S., Müller, R., and Lingens, F. (1992). Purification and some properties of 2-halobenzoate 1,2-dioxygenase, a two-component enzyme system from Pseudomonas cepacia 2CBS. J Bacteriol 174, 279-290.

Foster, C.E., Bianchet, M.A., Talalay, P., Zhao, Q., and Amzel, L.M. (1999). Crystal structure of human quinone reductase type 2, a metalloflavoprotein. Biochemistry 38, 9881-9886.

Gorman, J., and Shapiro, L. (2005). Crystal structures of the tryptophan repressor binding protein WrbA and complexes with flavin mononucleotide. Protein Sci 14, 3004-3012.

Ito, K., Nakanishi, M., Lee, W.C., Sasaki, H., Zenno, S., Saigo, K., Kitade, Y., and Tanokura, M. (2006). Three-dimensional structure of AzoR from Escherichia coli. An oxidereductase conserved in microorganisms. J Biol Chem 281, 20567-20576.

Lewis, D.F.V. (2001). Guide to Cytochromes P450: Structure and Function (New York, US, Taylor and Francis).

Li, R., Bianchet, M.A., Talalay, P., and Amzel, L.M. (1995). The threedimensional structure of $\mathrm{NAD}(\mathrm{P}) \mathrm{H}$ :quinone reductase, a flavoprotein involved in cancer chemoprotection and chemotherapy: mechanism of the two-electron reduction. Proc Natl Acad Sci U S A 92, 8846-8850.

Lichtenstein, G.R., Kamm, M.A. (2008). Review article: 5-aminosalicylate formulations for the treatment of ulcerative colitis-methods of comparing release rates and delivery of 5-aminosalicylate to the colonic mucosa. Aliment Pharmacol Ther 28, 663-673.

Liger, D., Graille, M., Zhou, C.Z., Leulliot, N., Quevillon-Cheruel, S., Blondeau, K., Janin, J., and van Tilbeurgh, H. (2004). Crystal structure and functional characterization of yeast YLR011wp, an enzyme with $\mathrm{NAD}(\mathrm{P}) \mathrm{H}-\mathrm{FMN}$ and ferric iron reductase activities. J Biol Chem 279, 34890-34897.

Liu, G., Zhou, J., Jin, R., Zhou, M., Wang, J., Lu, H., and Qu, Y. (2008). Enhancing survival of Escherichia coli by expression of azoreductase AZR possessing quinone reductase activity. Appl Microbiol Biotechnol 80, 409-416.

Liu, G., Zhou, J., Fu, Q.S., and Wang, J. (2009). The Escherichia coli azoreductase AzoR Is involved in resistance to thiol-specific stress caused by electrophilic quinones. J Bacteriol 191, 6394-6400.

Long, D.J., 2nd, Gaikwad, A., Multani, A., Pathak, S., Montgomery, C. A., Gonzalez, F.J., Jaiswal, A.K. (2002). Disruption of the NAD(P) $\mathrm{H}$ :quinone oxidoreductase 1 (NQO1) gene in mice causes myelogenous hyperplasia. Cancer Res 62, 3030-3036.

Nakanishi, M., Yatome, C., Ishida, N., and Kitade, Y. (2001). Putative ACP phosphodiesterase gene (acpD) encodes an azoreductase. J Biol Chem 276, 46394-46399.

Nissen, M.S., Youn, B., Knowles, B.D., Ballinger, J.W., Jun, S.Y., Belchik, S.M., Xun, L., and Kang, C. (2008). Crystal structures of $\mathrm{NADH}$ :FMN oxidoreductase $(\mathrm{EmoB})$ at different stages of catalysis. J Biol Chem 283, 28710-28720.

Patridge, E.V., and Ferry, J.G. (2006). WrbA from Escherichia coli and Archaeoglobus fulgidus is an $\mathrm{NAD}(\mathrm{P}) \mathrm{H}$ :quinone oxidoreductase. $\mathrm{J}$ Bacteriol 188, 3498-3506.

Peppercorn, M.A., and Goldman, P. (1972). The role of intestinal bacteria in the metabolism of salicylazosulfapyridine. J Pharmacol Exp Ther 181, 555-562.

Potterton, L., McNicholas, S., Krissinel, E., Gruber, J., Cowtan, K., Emsley, P., Murshudov, G.N., Cohen, S., Perrakis, A., and Noble, M. (2004). Developments in the CCP4 molecular-graphics project. Acta Crystallogr D Biol Crystallogr 60, 2288-2294.

Poulos, T.L., Finzel, B.C., Gunsalus, I.C., Wagner, G.C., and Kraut, J. (1985). The 2.6-A crystal structure of Pseudomonas putida cytochrome P-450. J Biol Chem 260, 16122-16130.

Roosild, T.P., Castronovo, S., Miller, S., Li, C., Rasmussen, T., Bartlett, W., Gunasekera, B., Choe, S., and Booth, I.R. (2009). KTN (RCK) domains regulate $\mathrm{K}^{+}$channels and transporters by controlling the dimer-hinge conformation. Structure 17, 893-903.

Ryan, A., Laurieri, N., Westwood, I., Wang, C.J., Lowe, E., and Sim, E. (2010). A novel mechanism for azoreduction. J Mol Biol 400, 24-37.

Sandy, J., Mushtaq, A., Holton, S.J., Schartau, P., Noble, M.E., and $\mathrm{Sim}, \mathrm{E}$. (2005). Investigation of the catalytic triad of arylamine Nacetyltransferases: essential residues required for acetyl transfer to arylamines. Biochem J 390, 115-123.

Savli, H., Karadenizli, A., Kolayli, F., Gundes, S., Ozbek, U., and Vahaboglu, H. (2003). Expression stability of six housekeeping genes: A proposal for resistance gene quantification studies of Pseudomonas aeruginosa by real-time quantitative RT-PCR. J Med Microbiol 52, 403 408.

Sherma, J.F. B. (2003). Handbook of thin layer chromatography, 3rd edn (New York, Marcel Dekker).

Shore, J. (1995). Dyeing with reactive dyes. In cellulosics dyeing, J. Shore, ed. (Manchester, UK, The Alden Press).

Sinclair, J.C., Sandy, J., Delgoda, R., Sim, E., and Noble, M.E. (2000). Structure of arylamine $\mathrm{N}$-acetyltransferase reveals a catalytic triad. Nat Struct Biol 7, 560-564.

Sollner, S., Nebauer, R., Ehammer, H., Prem, A., Deller, S., Palfey, B. 
A., Daum, G., and Macheroux, P. (2007). Lot6p from Saccharomyces cerevisiae is a FMN-dependent reductase with a potential role in quinone detoxification. FEBS J 274, 1328-1339.

Sollner, S., Deller, S., Macheroux, P., and Palfey, B.A. (2009a). Mechanism of flavin reduction and oxidation in the redox-sensing quinone reductase Lot6p from Saccharomyces cerevisiae. Biochemistry 48, 8636-8643.

Sollner, S., Schober, M., Wagner, A., Prem, A., Lorkova, L., Palfey, B. A., Groll, M., and Macheroux, P. (2009b). Quinone reductase acts as a redox switch of the $20 \mathrm{~S}$ yeast proteasome. EMBO Rep 10, 65-70.

Sparla, F., Tedeschi, G., Pupillo, P., and Trost, P. (1999). Cloning and heterologous expression of $\mathrm{NAD}(\mathrm{P}) \mathrm{H}$ :quinone reductase of Arabidopsis thaliana, a functional homologue of animal DT-diaphorase. FEBS Lett 463, 382-386.

Stolz, A. (2001). Basic and applied aspects in the microbial degradation of azo dyes. Appl Microbiol Biotechnol 56, 69-80.

Tomasz, M., and Palom, Y. (1997). The mitomycin bioreductive antitumor agents: cross-linking and alkylation of DNA as the molecular basis of their activity. Pharmacol Ther 76, 73-87.

Töwe, S., Leelakriangsak, M., Kobayashi, K., Van Duy, N., Hecker, M., Zuber, P., and Antelmann, H. (2007). The MarR-type repressor MhqR (YkvE) regulates multiple dioxygenases/glyoxalases and an azoreductase which confer resistance to 2-methylhydroquinone and catechol in Bacillus subtilis. Mol Microbiol 66, 40-54.

Wang, C.J. (2008). Characterisation of azoreductases from Pseudo- monas aeruginosa In Pharmacology (Oxford, University of Oxford), pp. 229.

Wang, C.J., Hagemeier, C., Rahman, N., Lowe, E., Noble, M., Coughtrie, M., Sim, E., and Westwood, I. (2007). Molecular cloning, characterisation and ligand-bound structure of an azoreductase from Pseudomonas aeruginosa. J Mol Biol 373, 1213-1228.

Wang, C.J., Laurieri, N., Abuhammad, A., Lowe, E., Westwood, I., Ryan, A., and Sim, E. (2010a). Role of Tyrosine 131 in the active site of paAzoR1, an azoreductase with specificity for the inflammatory bowel disease pro-drug balsalazide. Acta Crystallogr Sect F Struct Biol Cryst Commun 66, 2-7.

Wang, X., Jia, J., and Wang, Y. (2010b). Electrochemical degradation of reactive dye in the presence of water jet cavitation. Ultrason Sonochem 17, 515-520.

Ye, J., Yang, H.C., Rosen, B.P., and Bhattacharjee, H. (2007). Crystal structure of the flavoprotein ArsH from Sinorhizobium meliloti. FEBS Lett 581, 3996-4000.

You, S.J., Tseng, D.H., Ou, S.H., and Chang, W.K. (2007). Performance and microbial diversity of a membrane bioreactor treating real textile dyeing wastewater. Environ Technol 28, 935-941.

Zhang, J., Sun, Z., Li, Y., Peng, X., Li, W., Yan, Y. (2009). Biodegradation of $\mathrm{p}$-nitrophenol by Rhodococcus sp. CN6 with high cell surface hydrophobicity. J Hazard Mater 163, 723728. 